\title{
The Effect of Targeted Beta Blocker Use in Trauma Patients Admitted to the Intensive Care Unit
}

\author{
Akella Chendrasekhar*, Shanelle Hodge, Jakey Patwari, Vladimir Rubinshteyn, Loren Harris \\ Department of Surgery, Richmond University Medical center, 355 Bard Ave, Staten Island, USA
}

\begin{abstract}
Beta-blockers are used to reduce heart rate (HR) and thereby reduce myocardial oxygen demand. Current guidelines include a recommendation for the use of beta-blockers titrated to a target $\mathrm{HR}$ of 60 to 70 beats per minute (bpm) in certain populations; however, the value of this intervention has not yet been studied in the trauma population. Our objective was to determine whether beta-blockers used to titrate heart rate to $60-70 \mathrm{bpm}$ within the first 24 hours of intensive care unit (ICU) admission affect outcome in trauma patients.
\end{abstract}

We retrospectively examined the trauma registry to identify patients admitted to the ICU of an urban level-I trauma center over the course of January to December 2013; medical records were further reviewed to identify those who received treatment with beta-blockers. Data was collected on patient demographics, maximum/minimum HR within 24 hours of admission, achievement of target HR (60-70 bpm), type of beta-blocker administered, length of stay (LOS), injury severity score (ISS), and survival to discharge. Patients were stratified into two groups based upon whether target HR was achieved at least once during the first 24 hours of ICU admission. Statistical analysis was performed using chi-square analysis or t-test as appropriate.

A total of 208 patients, 65 females and 143 males with an average age of $59.3 \pm 19.3$ years, were included. The majority of patients $(88.9 \%)$ were treated with metoprolol while the rest received atenolol, labetalol, or carvedilol. Eighty-six patients reached target $\mathrm{HR}$ on the first day of ICU admission. Heart rate ranged from 48 to $150 \mathrm{bpm}$; maximum HR was significantly lower in the target HR achieved group ( $82 \pm 2.4 \mathrm{vs} 99.9 \pm 2.8 \mathrm{bpm}, \mathrm{p}<0.001)$. There was no significant difference between the amount of injury sustained by the target HR achieved (ISS $14.2 \pm 1.9$ ) and target HR not achieved (ISS $15.9 \pm 1.9$ ) groups. Achievement of target HR was associated with a shorter LOS (5.8 $\pm 1.3 \mathrm{~d}$ vs. $10.5 \pm 2.3 \mathrm{~d}$, p-value 0.002$)$ and increased likelihood of survival (OR 5.02, 95\%CI 1.67-15.2, $\mathrm{p}=0.004)$.

Keywords: Beta blocker; Trauma; Intensive care unit; Statistical analysis

\section{INTRODUCTION}

Trauma patients treated with beta-blockers who achieved target HR within the first 24 hours of ICU admission have a shorter Length of stay (LOS) and are more likely to survive to discharge than their counter parts. Further prospective research is necessary to determine the validity of our findings.

Beta blockers are commonly used in the treatment of cardiovascular diseases and to reduce the risk of re-infarction and the related mortality after myocardial infarction [1]. They reduce heart rate (HR) and thereby myocardial oxygen consumption. The ACCF/ AHA Task Force notes that the available evidence suggests, "when possible and where indicated, beta blockers should be started days to weeks before elective surgery. The current recommendation is a goal HR is 60 to 80 beats per minute. $\beta$ blockers, if used, should be appropriately titrated throughout the preoperative, intraoperative, and postoperative period to achieve effective heart rate control, while avoiding frank hypotension and bradycardia.

The pathophysiological concept that $\beta$-blockers can decrease cardiac oxygen consumption has led several authors to investigate the role of $\beta$-blockers in critical illness. Critical illness is associated to systemic inflammatory response (SIRS) characterized by increased resting energy expenditure due to sympathetic activation and a hypermetabolic state [2]. Studies have suggested that Beta-blocker use is associated with reduced risk of death within 30 days in general ICU patients and that the mortality reducing effect was similar among medical and surgical ICU patients and was not restricted to patients admitted with cardiovascular diseases [3]. Multiple studies

${ }^{*}$ Correspondence to: Akella Chendrasekhar, Vascular Surgery, University Medical Center, Department of surgery, 355 Bard Ave, Staten Island, USA, Tel: +1-718-818-1234; E-mail: chendrasekharakella@gmail.com

Received: May 06, 2020; Accepted: May 25, 2020; Published: May 30, 2020

Citation: Chendrasekhar A, Hodge S, Patwari J, Rubinshteyn V, Harris L (2020) The Effect of Targeted Beta Blocker Use in Trauma Patients Admitted to the Intensive Care Unit. J Vasc Med Surg 8:3. doi: 10.35248/2329-6925.20.8.391.

Copyright: (C) 2020 Chendrasekhar A, et al. This is an open-access article distributed under the terms of the Creative Commons Attribution License, which permits unrestricted use, distribution, and reproduction in any medium, provided the original author and source are credited. 
have shown a significant reduction in perioperative myocardial infarction and death from cardiac causes with the institution of perioperative beta blockade. These studies do not address target of therapy, simply starting $\beta$ blockers was documented.

Current guidelines include a recommendation for the usage of beta-blockers titrated to a target HR of 60 to 70 beats per minute (bpm) in certain populations [4]. However, the value of this intervention has not yet been studied in the trauma population. Our objective is to determine whether beta-blockers when titrated to a heart rate to $60-70 \mathrm{bpm}$ within the first 24 hours of intensive care unit (ICU) admission affect outcome in trauma patients that were on beta blockers at home prior to the injury which brought them to the ICU. Our hypothesis was that where beta blocker use was optimal (dosage titrated so that heart rate reached a target of $60-80$ beats per minute) during the first 24 hours, outcomes in these patients are improved.

\section{METHODS}

We retrospectively examined the trauma registry at our ACS verified level 1 trauma center to identify patients admitted to the ICU over the course of 2 years. Medical records were further reviewed to identify Trauma patients that were on beta blockers at home prior to injury, who subsequently were started on beta blockers at entry into the ICU. Data was collected on patient demographics, maximum/minimum HR within 24 hours of admission, achievement of target HR (60-70 bpm), type of betablocker administered, length of stay (LOS), injury severity score (ISS), and survival to discharge. Patients were stratified into two groups based upon whether or not target HR was achieved at least once during the first 24 hours of ICU admission. Data were analyzed using one-way analysis of variance (ANOVA) using a commercially available statistical analysis software package (JMP $14.0^{\circledR}$, SAS Institute). As this was a retrospective data analysis using de-identified data, we sought and obtained exemption from our institutional review board for the study of human subjects.

\section{RESULTS}

A total of 208 patients were selected 65 females and 143 male, with an average age of $59.3 \pm 19.3$ years were included. Majority of patients $(88.9 \%)$ were treated with metoprolol while the rest received atenolol (5.8\%), labetalol(3.4\%), or carvedilol (1.9\%). Eighty-six patients (41\%) reached target HR on the first day of ICU admission (Figures 1-3).

\section{Pts who reached target}

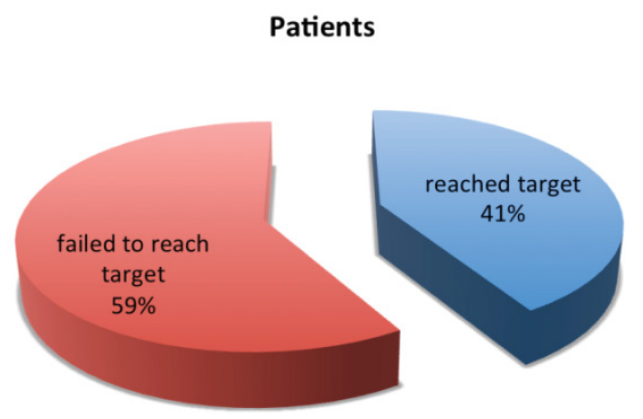

Figure 1: Heart rate ranged from 48 to $150 \mathrm{bpm}$; maximum HR was significantly lower in the target HR achieved group ( $82 \pm 2.4$ vs $99.9 \pm 2.8$ bpm, $\mathrm{p}<0.001$ ).

\section{Maximum HR during first 24 hours in}

ICU

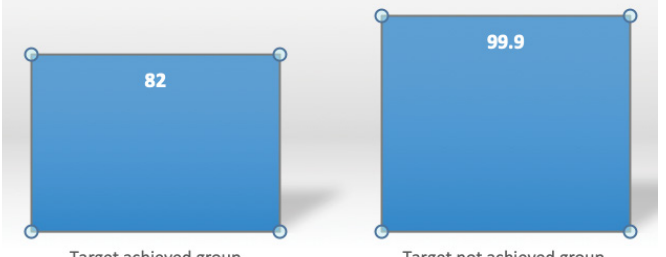

Target achieved group

Target not achieved group

Figure 2: There was no significant difference between the amount of injury sustained by the target HR achieved (ISS $14.2 \pm 1.9$ ) and target HR not achieved (ISS $15.9 \pm 1.9$ ) groups.

\section{Hospital Length of Stay}

$\mathrm{P}=0.002$

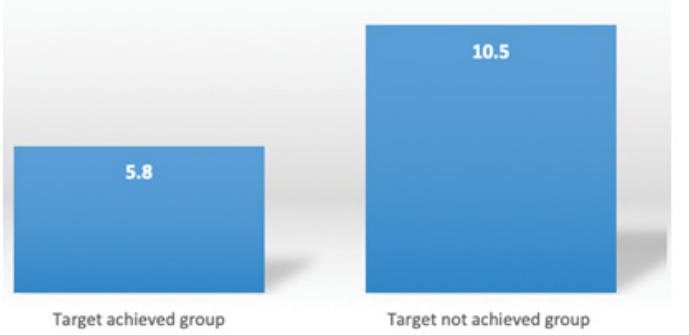

Figure 3: Achievement of target $\mathrm{HR}$ was associated with a shorter LOS $(5.8 \pm 1.3 \mathrm{~d}$ vs. $10.5 \pm 2.3 \mathrm{~d}$, p-value 0.002$)$ and increased likelihood of survival (OR 5.02, 95\%CI 1.67-15.2, $\mathrm{p}=0.004)$.

\section{DISCUSSION}

Significant Trauma in most cases is associated with systemic inflammatory response syndrome (SIRS) [5,6]. Specific trauma populations such as patients with traumatic brain injury have been shown to have improved outcomes and survival with beta blocker use [5]. The use of beta blockers in other traumatic injury has shown reduced mortality [6]. However, these studies did not document the targeted effectiveness of the beta blockers in achieving specific physiologic goals. The ability of our target achieved group of patients to mitigate the tachycardia related to the SIRS response would suggest that there is an inverse relationship between HR and survival. Our study shows that achieving target HR within the first 24 hours in patients on $\beta$ blockers is associated with improved outcomes. It is unclear whether this is due to a higher sympathetic tone in the control group or less responsiveness or a failure of titration of therapy. Limitations of our study were that it was retrospective in nature. We looked for documentation of at least 1 instance where target goal was reached, but this would not account for the entirety of the first 24 hours after injury. As this was a retrospective study we did not know whether the ICU team targeted a HR between $60-70$ or just empirically placed the patients on an arbitrary dose of beta-blockers. An informal survey of our intensivists would suggest that the empiric dose was selected based on previous dosage the patient was getting at home or arbitrary dosing without attempt to physiologically target a heart rate.

\section{CONCLUSION}

The maximal HR in this group of patients was significantly lower in patients that were able to reach our HR goal. This suggests that the SIRS response in the target achieved group may have been less or they may have had a better dose response to the beta blockers. Other potential confounding characteristics like cardiac co-morbidities 
were not included in data. In conclusion, trauma patients treated with beta-blockers who achieved target HR of 60-70 bpm within the first 24 hours of ICU admission have a shorter LOS and are more likely to survive to discharge than their counterparts. Further prospective research is necessary to determine the validity of our findings.

\section{REFERENCES}

1. Sanfilippo F, Santonocito C, Foex P. Use of beta-blockers in non-cardiac surgery: an open debate. Minerva Anestesiologica. 2014;80(4):482-494

2. Coppola S, Froio S, Chiumello D, Beta-blockers in critically ill patients: from physiology to clinical evidence. Critical Care. 2015;19:119.

3. Christensen S, Johansen MB, Tonnesen E, Larsson A, Pedersen L, Lemeshow S, et al. Preadmission beta-blocker use and 30-day mortality among patients in intensive care: a cohort study. Critical Care. 2011;15(2):R87.
4. Fleisher LA, Beckman JA, Brown KA, Calkins H, Chaikof EL, Fleischmann KA, et al. ACCF/AHA focused update on perioperative beta blockade incorporated into the ACC/AHA 2007 guidelines on perioperative cardiovascular evaluation and care for noncardiac surgery: a report of the American college of cardiology foundation/ American heart association task force on practice guidelines. Circulation. 2009;120(21):e169-276.

5. Ley EJ, Leonard SD, Barmparas G, Dhillon NK, Inaba K, Salim A, et al.. Beta blockers in critically ill patients with traumatic brain injury: Results from a multicenter, prospective, observational American Association for the Surgery of Trauma study. J Trauma Acute Care Surg. 2018;84(2):234-244.

6. Hendrick LE1, Schroeppel TJ, Sharpe JP, Alsbrook D, Magnotti LJ, Weinberg JA, et al. Impact of Beta-Blockers on Nonhead Injured Trauma Patients. Am Surg. 2016;82(7):575-579. 